\title{
Climatological features and trends of extreme precipitation during 1979-2012 in Beijing, China
}

\author{
Z. X. Xu${ }^{1,2}$ and Q. $\mathrm{Chu}^{1,2}$ \\ ${ }^{1}$ Key Laboratory of Water and Sediment Sciences, Ministry of Education, College of Water Sciences, \\ Beijing Normal University, Beijing, 100875, China \\ ${ }^{2}$ Joint Center for Global Change Studies, Beijing, 100875, China
}

Correspondence to: Z. X. Xu (zongxuexu@vip.sina.com)

Received: 15 April 2015 - Accepted: 15 April 2015 - Published: 11 June 2015

\begin{abstract}
In this study, three kinds of hourly precipitation series with the spatial resolution of $0.1^{\circ}$ are used to analyze the climatological features and trends of extreme precipitation during the period of 1979-2012 in Beijing, China. The results show that: (1) the spatial distribution of median annual precipitation, with a range from 500 to $825 \mathrm{~mm}$, is similar to that of local topography, which increases from the northwest to the southeast. Taking the urban area as a centre, the inter-annual precipitation in the Beijing area displays an outward decreasing tendency at the maximum rate of $125 \mathrm{~mm}$ per decade $\left(125 \mathrm{~mm} \times 10 \mathrm{a}^{-1}\right)$; (2) extreme precipitation amount, which accounts for $40-48 \%$ of total precipitation amount, has a similar spatial distribution to average annual precipitation; (3) the spatial distribution of extreme precipitation days and threshold estimated as the upper 95 percentile are significantly different from that of extreme precipitation, with maximum values concentrated on the urban area and the eastern mountain area, and minimum values in northwest; (4) extreme precipitation days (Ex_pd95) show an opposite distribution to extreme precipitation threshold (Ex_pv95), indicating that areas with greater precipitation threshold may has less precipitation days, and vice versa; (5) an apparent spatiotemporal decreasing tendency is detected in extreme precipitation amount. The downward tendencies are also found in extreme precipitation threshold. Unlike Ex_pv95, in most of the study area, Ex_pd95 is virtually unchanged; (6) downward trends of extreme precipitation is slightly smaller than that of annual precipitation, and the reducing amplitude of north-eastern areas are much higher than the areas in the southwest.
\end{abstract}

\section{Introduction}

Extreme weather and climatic events have drawn broader concerns during past years, particularly on the regional and local scales. It has been recognized that changes in extreme events are more likely to cause damages for human lives and their properties than gradual changes (Bonsal et al., 2001). In order to obtain a better understanding of potential risks for decision making in terms of societal adaptation to future climate change, the detection and attribution of past changes become increasingly significant (Madsen et al., 2014).

Extreme precipitation, which is regarded as the main factor contributing to water security, reflects the homogeneity of temporal and spatial distribution of precipitation. Extreme precipitation indices, such as extreme precipitation threshold, extreme precipitation days, extreme precipitation amount, are widely used to assess the variations of extremes in several studies (You et al., 2014). As an identical classification of extremes is not comparable among the areas with greatly varying climates, empirical ranking methods are recommended to determine the extreme threshold at different percentiles. Therefore, the formula introduced by Beard (1943) has come into wide use because it is more suitable for studies on the changing climate extremes (Folland and Anderson, 2002).

There have been plenty of studies on the analysis for variations and trends of extreme precipitation over global or regional scales. With respect to extreme precipitation, most of studies are based on in situ observations or large scaled gridded data downscaled from climate models (Koteswara et al., 2014; Li et al., 2015). In the local area, both the length of the 


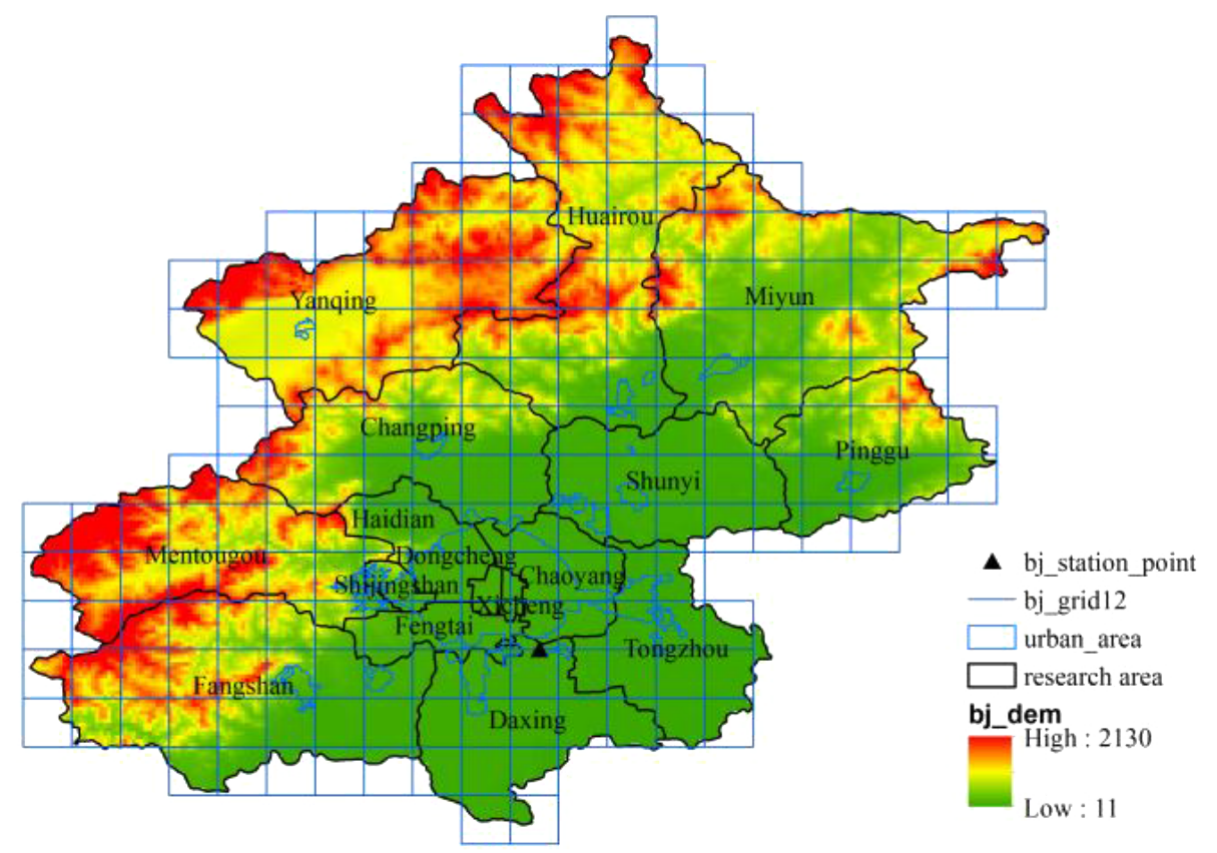

Figure 1. Map of Study Area. (The square grid represents the spatial resolution of the assimilated data, which covers 205 grids in the whole area. The areas in the northern area surrounded by the blue line stand for the urban areas. Elevation is also shown in this figure.)

data series and the spatial representative are limited due to the finite number of long-term observational stations. An urgent demand occurs for high resolution datasets for extremes studies especially in the rapidly urbanized regions with insufficient data. Under this circumstance, data assimilation technique undergoes a rapid development. It supplies an alternative way to study the impact of climate changes in these kinds of areas.

Beijing, the capital of China, has experienced tremendous changes due to the accelerated development of socioeconomics and the rapid expansion of population during the past fifty years. However, negative consequences, such as sever water scarcity, serious floods and urban water-logging, are all along with the rapid growth of economics and urbanization. Therefore, accurate quantifications of recent changes in extreme precipitation can be benefit to clarify the mechanism of climate change and enhance decision-making for sustainable development of water resources and environment protection in Beijing.

Due to the fact that surface precipitation changes exhibit obvious regional characteristics, few temporal and spatial studies with higher resolution data have been made so far in Beijing. The main objective of this study is to: (1) analyze the tempo-spatial variability of the annual extreme precipitation based on assimilated datasets with high resolution in Beijing; (2) qualitatively indicate the local-scale effects on extreme precipitation, such as topography, urbanization and local climate. Jenkinson's ranking formula and Theil-Sen Estimator are employed in this study. The findings will probably contribute to reduce uncertainties on floods and droughts induced by the variations of extreme precipitation.

\section{Study area description}

Beijing, the capital of the People's Republic of China, is composed of 16 districts, with most of the urban areas lying in the western area. It is located at $39^{\circ} 26--41^{\circ} 03^{\prime} \mathrm{N}$ and $115^{\circ} 25^{\prime}-117^{\circ} 30^{\prime} \mathrm{E}$ with an area of $16410.54 \mathrm{~km}^{2}$ (Beijing Statistics Bureau, 2010), $68 \%$ of which is mountain areas. It lies on the northwestern border of the North China Plain, surrounded by Taihang Mountain on the west and Yan Mountain on the north and northeast. Terrain tilts from northwest to southeast over the whole area. Elevation varies significantly $(60-2303 \mathrm{~m})$ in mountian areas; while it changes sligtly in Plain areas, with values from 10 to $60 \mathrm{~m}$, as shown in Fig. 1 .

The city is in the semi-humid warm continental monsoon climate zone. This place experiences four distinct seasons, with a cold and dry winter accompanied by northward wind blowing from high-latitude area, while a hot and wet summer because of the east-southeast toward airflow from the southern Pacific Ocean and the Indian Ocean. Due to the interaction of these cold and hot airflows, the precipitation is maily concentrated in summer, which accounts for $60-80 \%$ of total precipitation amount. 


\section{Data and method description}

In this study, a high resolution assimilated dataset (19792012) was used to analyze the variation of extreme precipitation. For each grid, Jenkinson's ranking formula was employed to estimate the 95th percentiles of daily precipitation distribution. The temporal and spatial characteristics and trends of surface annual extreme precipitation indices were then analyzed by Theil-Sen slope estimator method. A brief introduction on the dataset and methods are as follows.

\subsection{Data description}

In this study, three hourly assimilated datasets (1979-2012) with $0.1^{\circ} \times 0.1^{\circ}$ of spatial resolution were used to analyze the variations of extreme precipitation. These datasets used a global dataset produced by the Global Land Data Assimilation System (Rodell et al., 2004) as the background field when station observaitons are interpolated to grid points. Detailed data fusion technique may be found in $\mathrm{He}$ and Yang (2011). Simple quality control was also carried out to ensure that the time series is physically resonable by eliminating the data exceeding 3 standard deviations.

\subsection{Method description}

\subsubsection{Jenkinson empirical ranking formula}

According to Bonsal et al. (2001), daily precipitation for each year should be firstly ranked in ascending order $X_{1}$, $X_{2} \ldots X_{m} \ldots X_{n}$. The cumulative probability $\mathrm{P}$ that a random value is less than or equal to the rank of that value $X_{m}$ is then estimated by:

$P=(m-0.31) /(n+0.38)$.

This formula was proposed by Beard (1943) and presented in detail by Jenkinson (1977). It is proved by Folland and Anderson (2002) that this method performed as well as other empirical ranking formulas. But unlike other methods, Jenkinson's ranking method has no assumption on underlying distributions. That makes it more suitable to investigate the changing climate extremes, since knowledge of distribution form can rarely be obtained for those extremes.

\subsubsection{Theil-Sen estimator}

The Theil-Sen estimator is an unbiased estimator of the true slope in simple linear regression. For many distributions of the response error, this estimator has high asymptotic efficiency relative to least-squares. Estimators with low efficiency require more independent observations to attain the same sample variance of efficient unbiased estimators. Besides, it is more robust because it is much less sensitive to outliers: it can tolerate arbitrary corruption of up to $29.3 \%$ of the input data without degradation of the accuracy.

\section{Results analysis and discussion}

\subsection{Climatological features of extreme precipitation}

Figure 2 shows the spatial distribution of extreme precipitation indices in Beijing. The spatial distribution of median annual precipitation (PTV), with a range from 500 to $825 \mathrm{~mm}$, is opposite to that of local topography, which increases from the northwest to the southeast. Results by using Principal Component Analysis (PCA) method indicate that the local climate and topography are two main factors influencing the spatial distributions of precipitation.

Extreme precipitation threshold (Ex_pv95) calculated as the upper 95 percentile $\left(15.0-32.5 \mathrm{~mm} \mathrm{day}^{-1}\right)$ is slightly smaller than that estimated by You et al. (2014) in Beijing. It is likely due to the elimination of extremes by using standard deviation method. Extreme precipitation days (Ex_pd95) stand for the total time when daily precipitation is greater than Ex_pv95 in each year. As it can be seen from Fig. 1, Ex_pv95 presents an apparent opposite distribution to Ex_pd95, which means that areas with greater precipitation threshold may have less precipitation days and vice versa. The maximum Ex_pv95 appears at most of urban area and some districts in the northeast plain area, while with the least values in the north-western areas. Ex_pd95 in the piedmont areas is nearly 7 days, which is the largest value in the whole study area. This is not only related to the interaction of the warm and cold airflows influenced by the local monsoon climate, but also due to significant uplift effect of terrain, resulting in systematic intensification of precipitation process in these areas.

Extreme precipitation amount (Ex_ptv95) is defined as the total amount of daily precipitation which exceeds Ex_pv95. Figure 1 shows that extreme precipitation amount has a parallel spatial distribution to average annual precipitation, with maximum values concentrated on urban area and the eastern mountain area, and minimum values in the north-western area. It accounts for $40-48 \%$ of total precipitation amount within only 5 to 7 days, which indirectly suggests the inhomogeneous temporal characteristics of precipitation. It is worthwhile to notice that Ex_ptv95 of the urban areas occupies the largest proportion of total precipitation amount. Moreover, the total precipitation also has the maximum value of $825 \mathrm{~mm}$, displaying a strong feature of urban wet island effect. The reason for this is partly owing to the effect of urbanisation in terms of urban heat island, the obstacles of high-rise buildings and the increase of condensation nucleus.

Extreme precipitation intensity (Ex_pi95) is an important measurement of extreme precipitation, since larger Ex_pi95 implies higher risk caused by extreme precipitation. It is clear that the spatial characteristic of Ex_pi95 is similar to that of Ex_pv95, which suggests that areas with larger Ex_pv95 may experience heavy storm. The maximum values appear at the urban area and some north-eastern areas, which is just 

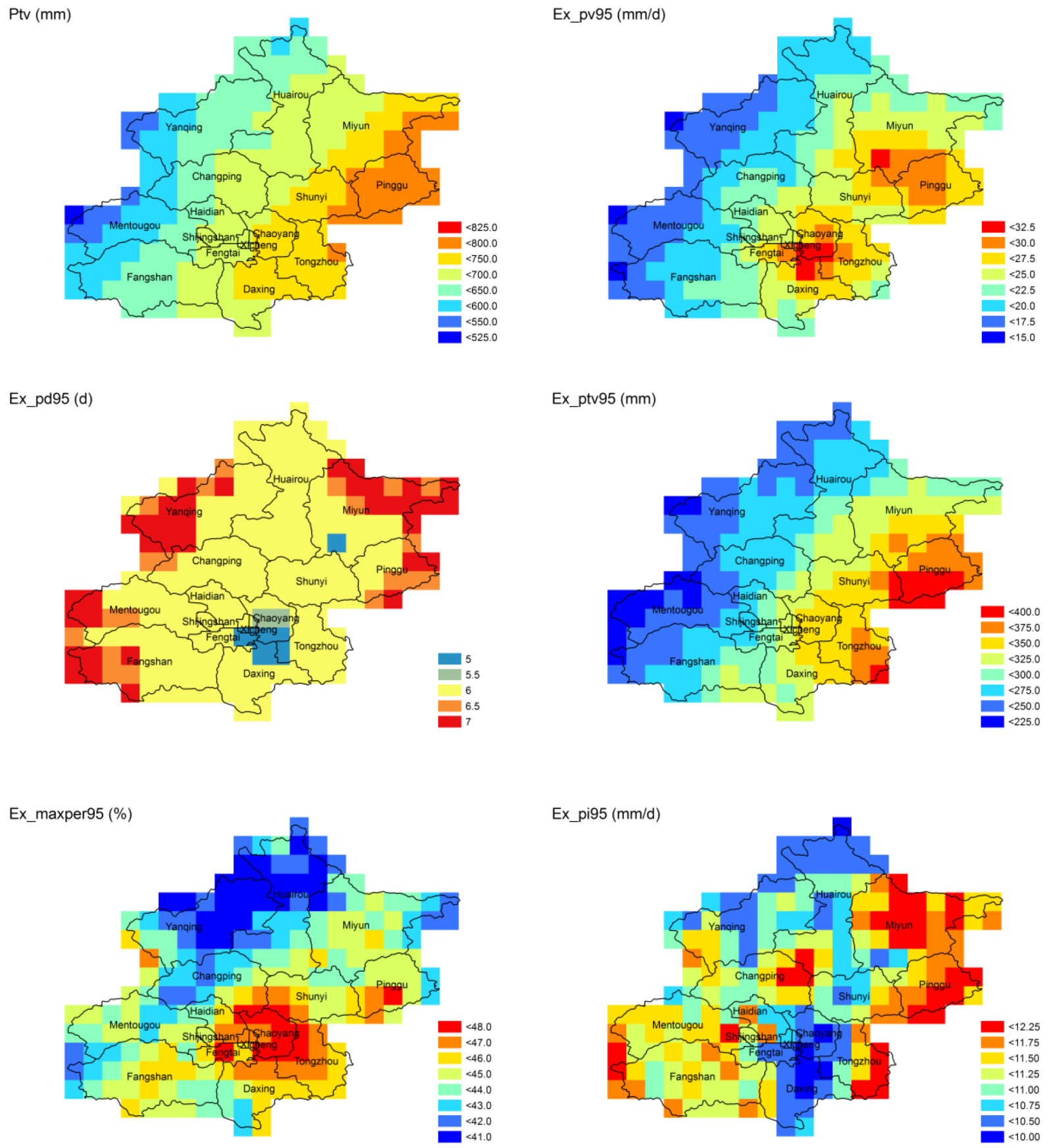

Figure 2. Climatological annual-median values of extreme precipitation based on $3 \mathrm{~h}$ gridded data during 1979-2012. (From the left to the right and from the up to the bottom, the figures referred to (a) median annual precipitation (PTV); (b) extreme precipitation threshold (Ex_pv95); (c) extreme precipitation days (Ex_pd95); (d) extreme precipitation amount (Ex_ptv95); (e) extreme precipitation proportion (Ex_maxper95); (f) extreme precipitation intensity (Ex_pi95) calculated at upper 95th percentile by Jenkinson's formula, respectively).

under $70 \mathrm{~mm} \mathrm{day}^{-1}$. This means that these regions should be paid more attention for the threat of extreme precipitation.

\subsection{Trends of extreme precipitation}

A significant downward trend can be found in both PTV and Ex_ptv95 in Beijing, with sharply decreasing rate (90$110 \mathrm{~mm} \times 10 \mathrm{a}^{-1}$ ) in the urban areas (see Fig. 3). Chu et al. (2015) found that the dramatic fall somehow related to the 


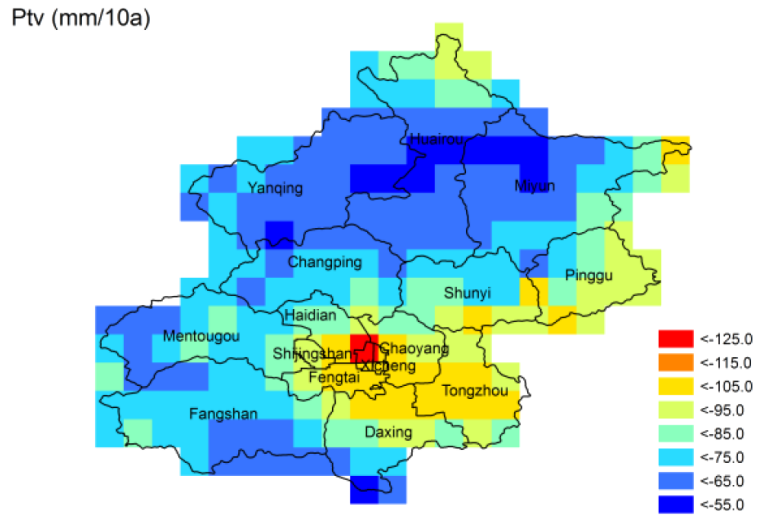

Ex_pd95 (d/10a)
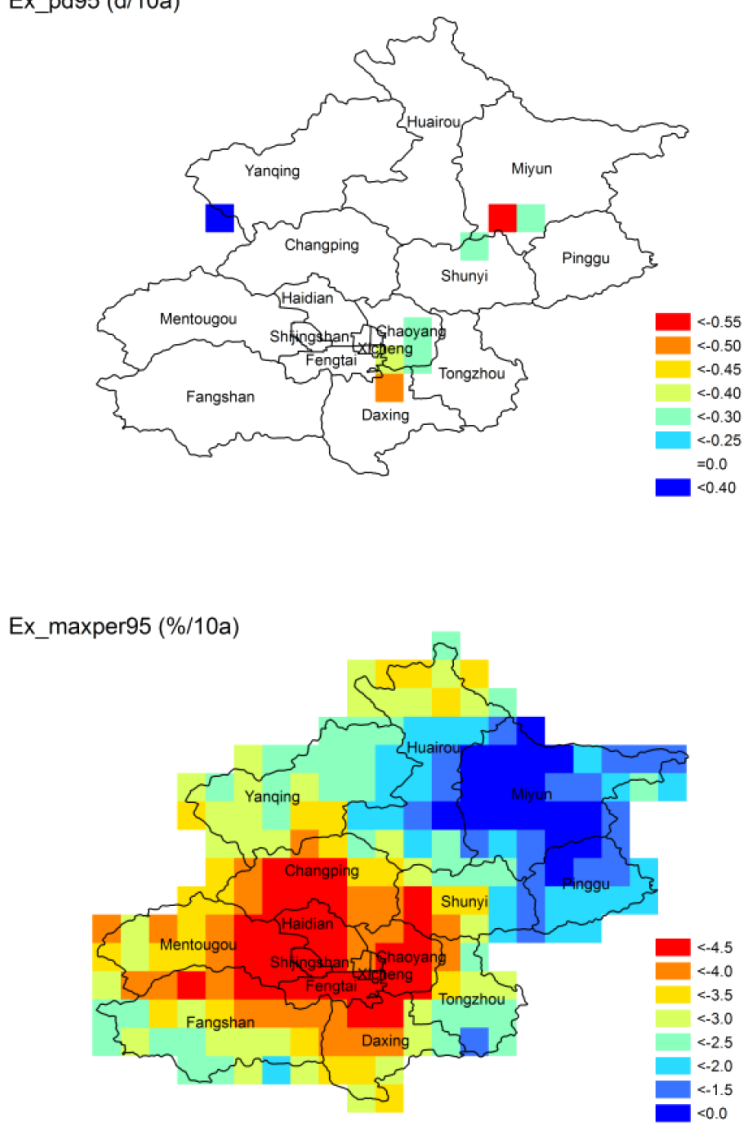

Ex_pv95 (mm/d·10a)

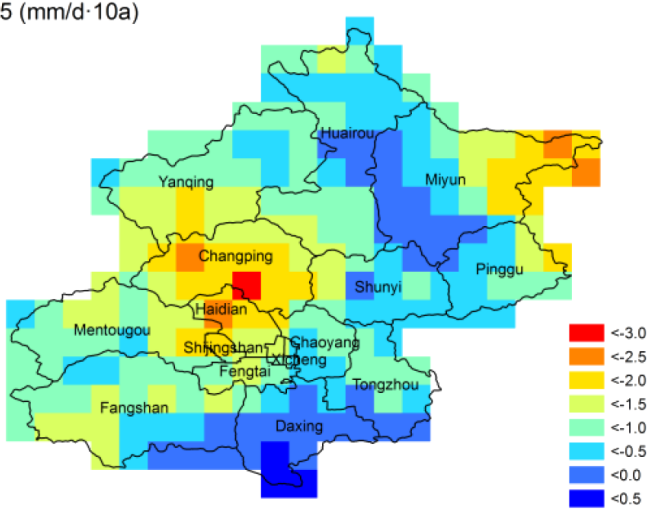

Ex_ptv95 (mm/10a)

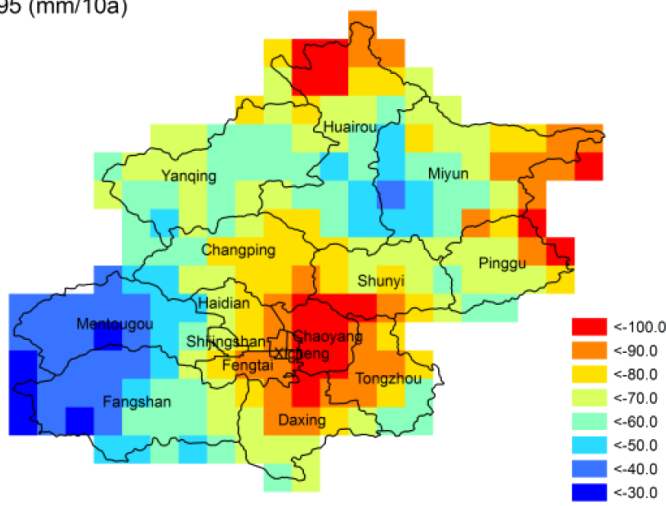

Ex_pi95 (mm/d·10a)

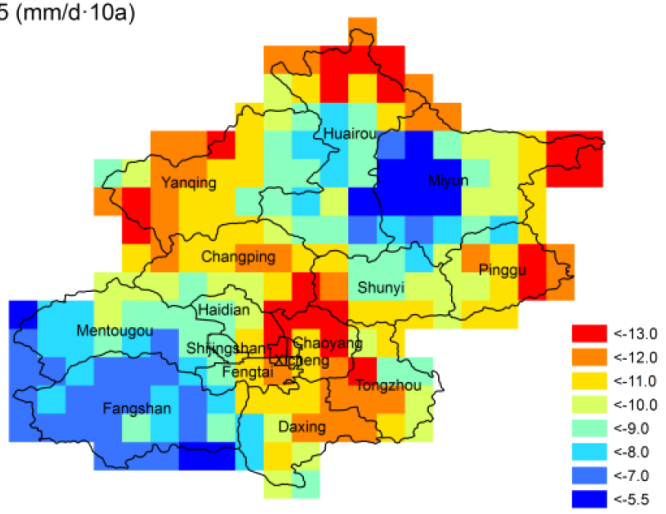

Figure 3. Trends of extreme precipitation based on $3 \mathrm{~h}$ gridded data during 1979-2012. (The variables are same as Fig. 2.)

rising temperature. Although the total water vapour amount increases because of the rise of evaporation, the capacity of atmosphere to hold water presents faster upward trend. An apparent spatial-temporal decreasing trend is detected in Ex_ptv95 values. The downward tendencies are also found in extreme precipitation threshold and days, which are more pronounced in Miyun and Mentougou districts.

According to the formula given by Jenkinson, the decrease of Ex_pv95 indicates reducing daily precipitation in- tensity, while the increase of Ex_pv95 represents a rise of daily precipitation. As it can be seen from Fig. 3, the northern and north-eastern districts have experienced an upward tendency in daily intensity, while the regions in north and east fell with the value of $3.0 \mathrm{~mm} \mathrm{day}^{-1} \times 10 \mathrm{a}^{-1}$. Unlike Ex_pv95, in most of the study area, Ex_pd95 is virtually unchanged, the decreasing amplitude of which is less than 0.55 day $\times 10 \mathrm{a}^{-1}$. These slight changes are detected in the 
areas where Ex_pv95 increased a lot, which leads to significant decrease of Ex_ptv95.

Compared the trends of PTV with that of Ex_ptv95, it is clear that the downward rate of PTV is nearly $30 \mathrm{~mm} \times 10 \mathrm{a}^{-1}$ greater than the rate of Ex_ptv95. On one hand, it suggests that Ex_ptv95 contributes the largest part in PTV. On the other hand, the proportion of Ex_ptv95 (Ex_maxper95) varied slightly during this period, indicating that the risk of extreme precipitation was still high, especially in the areas with the increase of Ex_pv95.

\section{Conclusions}

1. The spatial distribution of median annual precipitation increases from the northwest to the southeast. Results obtained by using Principal Component Analysis (PCA) method indicate that the local climate and topography are two main factors influencing the spatial distributions of precipitation in Beijing.

2. Ex_pv95 presents an apparent opposite distribution to Ex_pd95, which means that areas with greater precipitation threshold may have shorter precipitation days. The maximum Ex_pv95 appears at most of urban areas and some districts in the northeast plain area. The piedmont areas have the largest Ex_pd95 because of the effect of local monsoon climate and significant uplift of terrain.

3. Ex_ptv95 has a similar spatial distribution to average annual precipitation, with maximum values concentrated on the urban area and the eastern mountain area. It accounts for $40-48 \%$ of total precipitation amount within only 5 to 7 days, which indirectly suggests the inhomogeneous temporal characteristics of precipitation.

4. The spatial characteristics of Ex_pi95 are similar to that of Ex_pv95, with the maximum values appearing at the urban area and some north-eastern areas. These areas may experience heavy storm since larger Ex_pi95 implies higher risk caused by extreme precipitation.

5. Significant downward trends are detected in both PTV and Ex_ptv95, with sharply decreasing rate (90$\left.110 \mathrm{~mm} \times 10 \mathrm{a}^{-1}\right)$ in urban areas. This dramatic decrease is partly because the rise of air temperature, which results in higher rising rate of the capacity of atmosphere to hold water than the total water vapour amount.

6. The northern and north-eastern districts have experienced an upward tendency in daily intensity, while the regions in north and east fell with the value of $3.0 \mathrm{~mm}$ day $^{-1} \times 10 \mathrm{a}^{-1}$. Unlike Ex_pv95, in most of the study area, Ex_pd95 is virtually unchanged.
7. Ex_ptv95 contributed the largest part in the decrease of PTV. However, the proportion of Ex_ptv95 (Ex_maxper95) varied slightly during this period, indicating that the risk of extreme precipitation was still high, especially in the areas with the increase of Ex_pv95.

Acknowledgements. This study was supported by the research project from Beijing Natural Science Foundation (No. 8141003). The authors thank the Data Assimilation and Modelling Centre for Tibetan Multi-spheres (DAM) for providing high resolution dataset of surface precipitation.

\section{References}

Beard, L. R.: Statistical analysis in hydrology, T. Am. Soc. Civ. Eng., 108, 1110-1121, 1943.

Beijing Statistics Bureau: Beijing Statistical Yearbook 2010, China Statistics Press, Beijing, 2010.

Bonsal, B. R., Zhang, X., Vincent, L. A., and Hogg, W. D.: Characteristics of daily and extreme temperatures over Canada, J. Climate, 14, 1959-1976, 2001.

Chu, Q., Xu, Z. X., Peng, D. Z., Yang, X. J., and Yang, G.: Trends of surface humidity and temperature during 1951-2012 in Beijing, China, in: Proceedings of the 3rd Remote Sensing and Hydrology Symposium, August 2014, Guangzhou, China, in press, 2015.

Folland, C. and Anderson, C.: Estimating changing extremes using empirical ranking methods, J. Climate, 15, 2954-2959, 2002.

He, J. and Yang, K.: China Meteorological Forcing Dataset, Cold and Arid Regions Science Data Center, Lanzhou, doi:10.3972/westdc.002.2014.db, 2011.

Jenkinson, A. F.: The analysis of meteorological and other geophysical extremes, Synoptic Climatology Branch, Meteorological Office, Bracknell, Berkshire, UK, 44 pp., 1977.

Koteswara, R. K., Patawardhan, S. K., Kulkarni, A., Kamala, K., Sabade, S. S., and Kumar, K. K.: Projected changes in mean and extreme precipitation indices over India using PRECIS, Global Planet. Change, 113, 77-90, 2014.

Li, J. F., Zhang, Q., Chen, Y. Q., and Singh, V. P.: Future joint probability behaviors of precipitation extremes across China: Spatiotemporal patterns and implications for flood and drought hazards, Global Planet. Change, 124, 107-122, 2015.

Madsen, H., Lawrence, D., Lang, M., Martinkova, M., and Kjeldsen, T. R.: Review of trend analysis and climate change projections of extreme precipitation and floods in Europe, J. Hydrol., 519, 3634-3650, 2014.

Rodell, M., Houser, P. R., Jambor, U., GottschalcK, J., Mitchell, K., Meng, C. J., Arsenault, K., Cosgrove, B., Radakovich, J., Entin, K., Walker, J. P., Lohmann, D., and Toll, D.: The Global Land Data Assimilation System, Am. Meteorol. Soc., 3, 381394, 2004.

You, H. L., Liu, W. D., and Ren, G. Y.: Variation characteristics of precipitation extremes in Beijing during 1981-2010, Clim. Environ. Res., 19, 69-77, 2014. 